\title{
EL PUENTE DEL MONTE EN AHIGAL (CÁCERES): AUTORÍA Y PROCESO CONSTRUCTIVO DE UNA JOYA SUMERGIDA EN LA ALTA EXTREMADURA
}

\author{
EL PUENTE DEL MONTE IN AHIGAL (CÁCERES): AUTHORSHIP \\ AND CONSTRUCTION PROCESS OF A SUBMERGED JEWEL IN \\ UPPER EXTREMADURA
}

\author{
FRANCISCO JAVIER CAMBERO SANTANO \\ Universidad de Extremadura
}

Recibido: 11/06/2019

Aceptado: 30/10/2019

RESUMEN

El río Alagón discurre, entre otros términos, por el de Ahigal, dividiendo el territorio en dos durante largos períodos. Para su paso se usaban barcas que, además de peligrosas, apenas suplían las necesidades de los viandantes. A finales del XIX, ante la necesidad de construir un puente, se llevó a cabo la obra del que sería conocido como Puente del Monte. Tradicionalmente, se ha creído que los vecinos de Ahigal costearon esta obra en su totalidad, pero no es del todo correcto, pues sería la Diputación de Cáceres quien cubriera, en parte, el importe del proyecto que atribuimos a D. Emilio $\mathbf{M}^{\mathrm{a}}$ Rodríguez y que fue inspeccionado por D. Vicente Paredes.

Ochenta años después, el puente perderá su función de ingeniería civil al quedar, por siempre, sumergido en las aguas del embalse de Valdeobispo.

Palabras clave: Puente del Monte, Emilio Ma Rodríguez, Vicente Paredes, arqueología pública, ingeniería civil, s. XIX. 


\section{ABSTRACT}

The river Alagón runs among other terms by Ahigal, dividing the territory in two for long periods. For it passage boats were used, that in addition to dangerous, as soon as they supplied the necessities of the pedestrians. At the end of the 19th century, faced with the need to build a bridge, the work of what would be known as Puente del Monte was carried out. Traditionally, it has been believed that the inhabitants of Ahigal paid for this work in its entirety, but it is not entirely correct, since Diputación de Cáceres would cover, in part, the amount of the project that we attributed to Emilio $\mathbf{M}^{\mathrm{a}}$ Rodríguez and that was inspected by Vicente Paredes Guillén.

Eighty years later, the bridge will lose its civil engineering function when it remains, forever, submerged in the waters of Valdeobispo Dam.

Keywords: Bridge of the Monte, Emilio $\mathrm{M}^{\mathrm{a}}$ Rodríguez, Vicente Paredes, public archaeology, hydraulic Engineering, 19th century.

“aun parece resonar todavía los ecos agonizantes

de los náufragos implorando socorro" 


\section{INTRODUCCIÓN.}

La aparición y desarrollo del ferrocarril en el s. XIX va a transformar las comunicaciones terrestres existentes hasta entonces. La desigualdad geográfica que la región extremeña presenta de norte a sur, dividida por los ríos Tajo y Guadiana, dará lugar a la construcción de numerosas obras ${ }^{1}$. Para ello, en Extremadura, serán necesarias la modernización y construcción de obras de ingeniería civil que ayuden a una mejor comunicación de las áreas rurales con aquellos puntos donde se fijarán las principales estaciones de tren para, por un lado, el paso del ferrocarril (ejemplo de ello, en la provincia, son los puentes del ferrocarril sobre el Tajo, en el término municipal de Garrovillas de Alconétar) y, por otro, la continuidad de los caminos vecinales y carreteras (como por ejemplo el Puente del Cardenal, en pleno Parque Nacional de Monfragüe, el Puente de Almaraz o el Puente del Monte), si bien son más numerosos que los primeros.

A principios del s. XX, ferrerías, batanes y molinos conformaron una red de pequeñas industrias que retomarían importancia con la llegada de la electricidad $^{2}$. En la década de 1960, la necesidad de producir más energía era latente y, junto a las políticas de regadío que se estaban implantando en Extremadura, los ríos empezaron a ser intervenidos para el aprovechamiento hidráulico de sus aguas. Esta circunstancia no fue favorable para aquel patrimonio que se encontraba cerca del cauce de aquellos, pues al entrar en acción la construcción de estos saltos, con grandes embalses y con sus centrales de pie de presa, serían desplazados a un segundo plano y, con el paso del tiempo, abandonados por diferentes motivos, como la inundación de los mismos ${ }^{3}$.

El embalse de Valdeobispo, al norte de la provincia de Cáceres, se comenzó a construir durante los últimos años de la década de 1950 y finalizaría en 1964. El motivo de su construcción estuvo directamente ligado a la de otro embalse, el de Gabriel y Galán. Al finalizar las obras de este, en 1961, observaron cómo el sistema hidráulico del río Alagón quedaba incompleto, por lo que era necesario el levantamiento de otra construcción que complementara la anterior. Esta presa, de tipo gravedad con vertedero por coronación y planta

1 Este trabajo ha sido realizado gracias a la financiación de la Fundación Fernando ValhondoCalaff.

2 GARCÍA ADÁN, J.C., "El aprovechamiento hidroeléctrico del río Tajo en la provincia de Cáceres en tiempo de la II República (1931 - 1936)" , Revista de Estudios Extremeños, n Extraordinario, Tomo LXXI, 2015, pp. $189-208$.

3 GARCÍA ADÁN, J.C., "Las aceñas del Tajo: aprovechamientos industriales en el tramo del Embalse de Alcántara” . En LOZANO BARTOLOZZÍ, M.M. Y MÉNDEZ HERNÁN, V., Paisajes modelados por el agua: entre el arte y la ingeniería, Mérida, Editora Regional de Extremadura, 2012, pp. 82 y ss. 
curva, tiene una altura máxima de $56 \mathrm{~m}$. y la longitud de coronación de $140 \mathrm{~m}$. $\mathrm{Al}$ igual que ocurriría con la mayoría de las presas, la empresa que recibió la concesión sería Hidroeléctrica Española ${ }^{4}$.

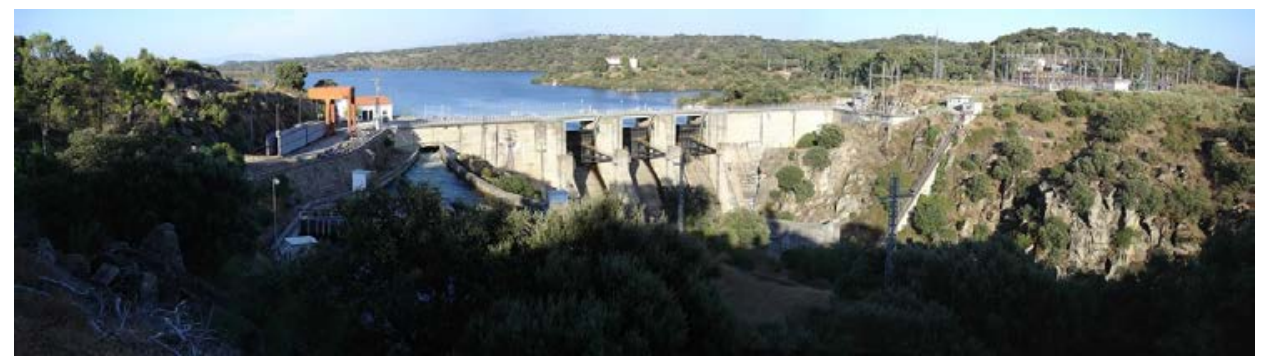

Figura 1. Panorámica de la Presa de Valdeobispo. Fuente: http://www.valdeobispo.net/Presa/Panoramical.html

Los pueblos más próximos a esta construcción se verían beneficiados en cuanto a los terrenos de regadío. En 1963, dieron comienzo la construcción de dos canales, uno por el margen derecho, de $65 \mathrm{~km}$, y otro por el izquierdo, de $90 \mathrm{~km}$. El primero domina los términos de Montehermoso, Galisteo, Guijo de Galisteo, Morcillo, Guijo de Coria, Coria, Calzadilla, Casas de Don Gómez y Casillas de Coria; mientras que el segundo domina los de Valdeobispo, Carcaboso, Aldehuela de Jerte, Galisteo, Plasencia, Oliva, Riolobos, Holguera, Torrejoncillo, Coria y Portage 5 .

Como en otros muchos casos en la provincia, parte del patrimonio quedó anegado bajo las aguas de los embalses, debido a que las políticas del momento buscaban generar riquezas a partir de la construcción de presas, pero no se tuvo en cuenta la riqueza material e inmaterial propia de la identidad de Extremadura que se iba a perder, como es el caso del Puente en estudio, donde Diputación de Cáceres invertiría una cuantía económica importante, o como el de otros tantos que quedaron sumergidos por no haberse propuesto un traslado de los mismos a tiempo ${ }^{6}$.

4 La Presa. Armando Pañero, 2006, [consulta 05-02-2019]. Disponible en: http://www.valdeobispo.net/Presa.html

5 BUENO HERNÁNDEZ, F., "El río Alagón: pasado, presente y futuro de sus obras hidráulicas” , Revista de Obras Públicas, no. 3.356, 1996, pp. 48 - 49.

6 CAMBERO-SANTANO, F.J., "Damaged-cultural landscapes by new cultural-landscapes. Transmission of knowledge of three underwater bridges in Tagus river" , En OOSTERBEEK, L.; GUDAUSKAS, R. Y CARON, L., Transdisciplinary contributions to Cultural Integrated Landscape Management. Mação: Instituto Terra e Memória, série Arkeos, nº 42. vol. 2, 2017, pp. 108 - 120. 


\section{EMILIO Ma RODRIGUEZ, POSIBLE ARQUITECTO DE LA OBRA.}

Aunque se desconocen los datos relativos a su infancia, Emilio M. ${ }^{\mathrm{a}}$ debió realizar sus estudios básicos en Salamanca, hasta que decidió formarse como arquitecto, motivo por el cual tuvo que desplazarse hasta Madrid. Para ello, cursó sus estudios en la Escuela Especial de Arquitectura de Madrid, creada en 1844, bajo la influencia de Ortiz y Villajos, Rodríguez Ayuso, Velázquez y Bosco, Adaro o Arbós 8 ; donde fue examinado y aprobado por pluralidad de votos en el ejercicio final de carrera, el día 8 de mayo de 1875, ante el tribunal de profesores, nombrado al efecto por el Claustro de dicha Escuela y, en consecuencia, fue declarado apto para obtener el título, que le fue expedido el día 24 de mayo del año anterior, con arreglo a las disposiciones vigentes ${ }^{9}$.

Tan solo un año más tarde, el día 15 de julio de 1876, Emilio remitiría desde Madrid toda la documentación necesaria para presentarse a una plaza de arquitecto municipal que se encontraba vacante en la ciudad de Cáceres. El 14 de septiembre del mismo año, fue nombrado arquitecto municipal de Cáceres mediante concurso con un sueldo de 3.000 pesetas $^{10}$, y tomaría posesión el 1 de octubre. En este cargo estaría hasta el 20 de noviembre de 1909 y no hasta el 21 de octubre de 1883 como aparece es su expediente personal. Un año después, en 1877, Emilio $\mathrm{M}^{\mathrm{a}}$ compaginaría su trabajo de arquitecto municipal con otros de mayor enjundia. El 16 de agosto obtendría el nombramiento de R. O. Arquitecto diocesano de Cáceres, puesto del que tomaría posesión el día 18 del mismo mes. Bajo este nombramiento realizaría alguna obra religiosa como por ejemplo, las intervenciones que llevó a cabo en la Iglesia de San Eugenio, en Aldea Moret.

Y si todo ello no fuera suficiente, el 9 de noviembre de 1877 fue nombrado y tomó posesión como arquitecto provincial.

7 Emilio Ma Rodríguez García, (1848-1916), nació en Salamanca, aunque autores como HURTADO, P., en Ayuntamiento y familias cacerenses, Cáceres, 1910, p. 729. lo sitúen, erróneamente, en Churriana, Málaga. Hijo de Vicente Rodríguez, natural de Cáceres, y de Manuela García Villanueva, vecina de Salamanca. Sus abuelos paternos eran Antonio Rodríguez, natural de Mérida y Petra Bejarano, de Cáceres; y sus abuelos maternos, Pedro García, natural de Pontevedra, y Juana López, de Salamanca. En ARCHIVO DIOCESANO DE SALAMANCA/PARROQUIAL (ADS), 417/7, 153v. San Juan de Barbalos, Salamanca.

8 LOZANO BARTOLOZZI, M. M.; CRUZ VILLALÓN, M. La arquitectura en Badajoz y Cáceres: del eclecticismo fin de siglo al racionalismo (1890-1940). Asamblea de Extremadura, 1995, p. 120.

9 ARCHIVO MUNICIPAL DE CÁCERES (AMCC) /55, negociado 20/660, 7/1909. Expediente de Emilio María Rodríguez, arquitecto municipal, solicita ser relevado de servicios inherentes a su cargo que durante años ha estado desempeñando. Cáceres, 1909.

10 ARCHIVO Y BIBLIOTECA DIPUTACIÓN PROVINCIAL DE CÁCERES /ADMINISTRACIÓN (ADPCC), Expediente personal de D. Emilio Rodríguez, de arquitecto provincial, 1877. 
Mientras desempeñaba su trabajo en los diferentes puestos, Rodríguez fue adquiriendo reputación y reconocimiento en el campo de la arquitectura; tanto es así que, el 27 de marzo de 1885, obtendría el nombramiento Real de director facultativo de las obras de restauración del Monasterio de Nuestra Señora de Guadalupe. Este cargo lo desempeñó hasta el 15 de abril de 1887. Pero estas funciones no fueron las únicas que desempeñó, también poseía servicios especiales, honores y títulos académicos.

En 1876, fue nombrado Individuo de la Comisión especial de evaluación y repartimiento de Cáceres. En 1877, vocal de la Junta del Censo General y vocal de la Junta provincial de Sanidad en diferentes bienios. En 1884, fue nombrado vocal de la Comisión Provincial de Reformas Sociales y, un año más tarde, en 1885, vocal de la Junta de Teatros y Espectáculos Públicos. En 1888, fue nombrado vocal de la Comisión Oficial creada en Cáceres para promover la concurrencia a la Exposición Universal de Barcelona. En 1905, obtuvo el nombramiento de Académico correspondiente de la de Bellas Artes y, por último, fue vocal nato de la Junta Provincial de Instrucción Pública y de la Comisión de Monumentos.

Rodríguez fallecería en la ciudad de Cáceres sin otorgar disposición testamentaria, siendo Antonio Rodríguez su único heredero, aunque su mujer disfrutaría del usufructo como marcaba la ley ${ }^{11}$.

\section{EL PUENTE DEL MONTE: ATRIBUCIÓN DE SU AUTORÍA A EMILIO MARÍA RODRÍGUEZ.}

En la provincia de Cáceres son escasos los municipios que, en los últimos años del período decimonónico y los primeros de la nueva centuria, cuenten con un Arquitecto Municipal. Eduardo Hervás en Cáceres y Trujillo, Vicente Paredes en Plasencia y Don Benito, o Sebastián Rebollar en Trujillo ${ }^{12}$ son algunos ejemplos de personalidades que desarrollarán sus obras municipales de manera coetánea a Rodríguez, al frente de trabajos en Cáceres y en pequeños municipios como arquitecto provincial.

Al no poseer el proyecto de obras del Puente del Monte, proponemos la atribución del mismo a Emilio $\mathrm{M}^{\mathrm{a}}$ Rodríguez, y no a Vicente Paredes como

11 ADPCC, Cédula n 5563, Clase 11 $11^{\text {a }}, 6$ de abril de 1916.

12 PIZARRO GÓMEZ, F.J., Arquitectura y urbanismo en Trujillo (siglos XVIII y XIX). Cáceres, Universidad de Extremadura, 1987. 
tradicionalmente se ha pensado ${ }^{13}$. Para ello, realizaremos una comparativa del puente con proyectos de otros realizados por él en el territorio cacereño que, coincidiendo en un corto período de tiempo, poseen unas características artísticas y arquitectónicas muy similares, y que fueron desarrollados en pequeños núcleos urbanos carentes de arquitecto municipal, siendo el arquitecto provincial el encargado de su realización.

Los proyectos de Rodríguez siempre se acompañaban de un expediente formado por la memoria, planos, pliego de condiciones facultativas y un presupuesto, que serían puestos a disposición del contratista que, ante cualquier duda, acudiría al arquitecto, a no ser que el ayuntamiento nombrara un director de obra, quien también podría ejercer como inspector para el desarrollo de las mismas, Vicente Paredes en el caso en estudio.

Entre los proyectos de puentes ideados por Emilio $\mathrm{M}^{\mathrm{a}}$ encontramos, entre otros, los trabajos de reparación y ampliación del puente sobre el río Magasca en el camino de la Cumbre a Trujillo ${ }^{14}$, o proyectos para la construcción de nueva planta, planeados en algunas localidades como Aliseda, donde se proyectó un puente sobre la Rivera Cagancha ${ }^{15}$, que no se llevó a cabo al decantarse por la opción más económica de un alcantarillado; otro para el molino de la iglesia y, por último, otro en el lugar conocido como el Tomadero ${ }^{16}$. En Arroyomolinos de Montánchez se proyectó el puente para el Arroyo del Pueblo ${ }^{17}$, en Pinofranqueado el Puente de los Ángeles ${ }^{18}$, en el actual Santiago de Alcántara uno sobre el regato Rivera-Aurela ${ }^{19}$ o, por último, en Villanueva de la Vera, el pontón sobre la garganta Gualtaminos ${ }^{20}$.

13 RABÁN, E., "El puente de Aygal" Revista Cultural Ahigal, no 62, julio - septiembre, 2015, pp. $11-14$.

14 Op. cit., PIZARRO GÓMEZ, F.J., Arquitectura y urbanismo en Trujillo...., pp. 226 - 231.

15 ARCHIVO MUNICIPAL DE ALISEDA (AMA), "Proyecto de un Puente sobre el regato llamado Cagancha” , Caja 539, 1883. 1883.

16 AMA, "Proyecto de puentes para el paso del Tomadero y molino de la Iglesia" , Caja 539,

17 ARCHIVO HISTÓRICO PROVINCIAL DE CÁCERES (AHPCC), "Proyecto de puente para el Arroyo del pueblo” , Legajo 3700/2, 1887.

18 ADPCC, "Proyecto para la construcción de un puente sobre el río de los Ángeles entre los términos Pinofranqueado y La Pesga y estudio para designar el punto más conveniente” , Signatura $05172,1881$.

19 AHPCC, "Proyecto de puente sobre el regato Rivera - Aurela en el camino de Santiago de Carbajo a Valencia de Alcántara", Legajo 3705/2, 1885.

20 AHPCC "Proyectos de obras municipales", Legajo 3708/4, 1889. 


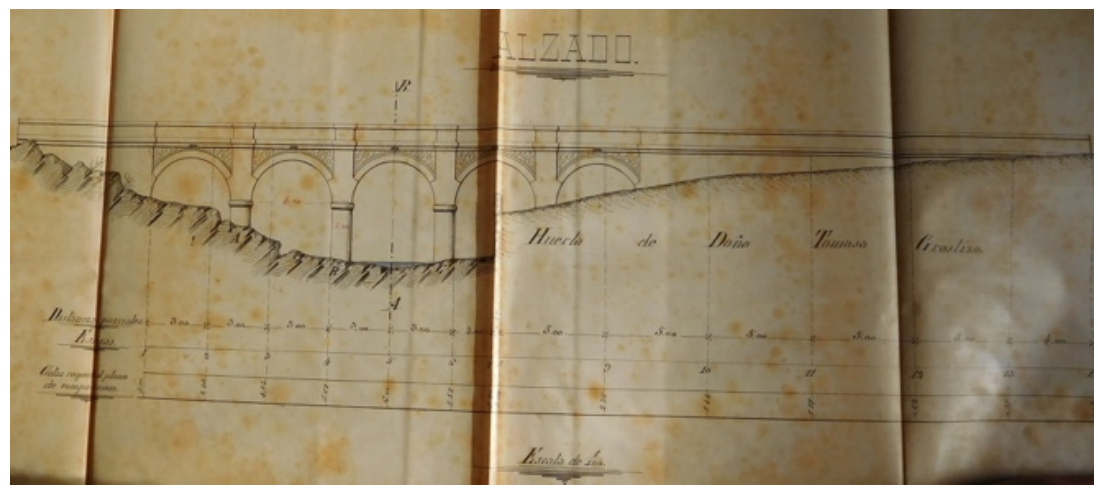

Figura 2. Proyecto de puente sobre la rivera Cagancha. Fuente: Archivo Municipal de Aliseda. Escala: 1:100

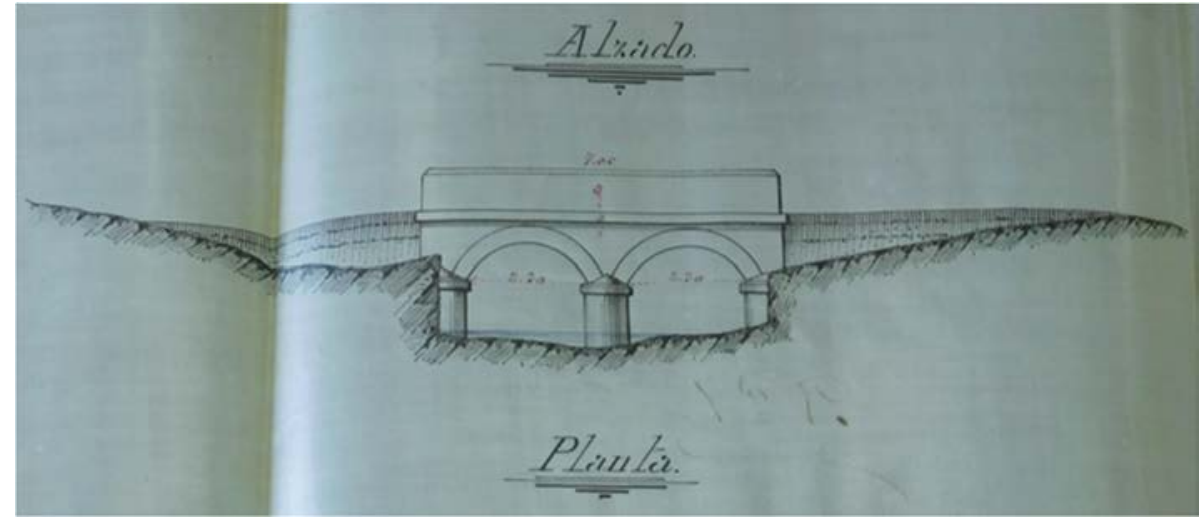

Figura 3. Proyecto de puente en Arroyomolinos de Montanchez. Fuente: Archivo Histórico Provincial de Cáceres. Escala: 1:100

Por norma general, el arquitecto proyectaba los puentes orientados en sentido normal a la dirección de las aguas y, teniendo en cuenta la línea marcada por el nivel de las crecidas, incidía los arcos.

El puente del Monte cuenta con nueve arcos rebajados, también llamados escarzanos o de círculo. Todos los puentes proyectados por el arquitecto tomaban esta característica, a excepción de una minoría de arcos de medio punto para los desagües o, como en el caso de Arroyomolinos de Montánchez, que se vio en la necesidad de proyectar el primero adintelado. 


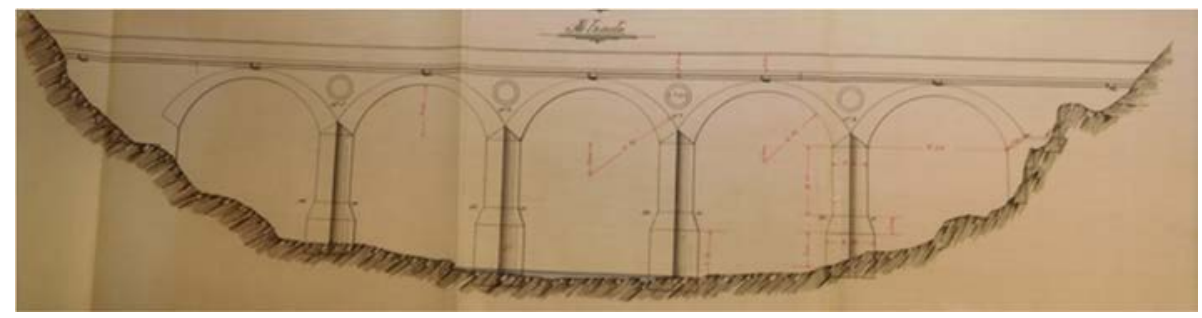

Figura 4. Proyecto de puente sobre el río de los Ángeles, Pinofranqueado y La Pesga. Fuente: Archivo y Biblioteca Diputación Provincial de Cáceres. Escala: 1:100

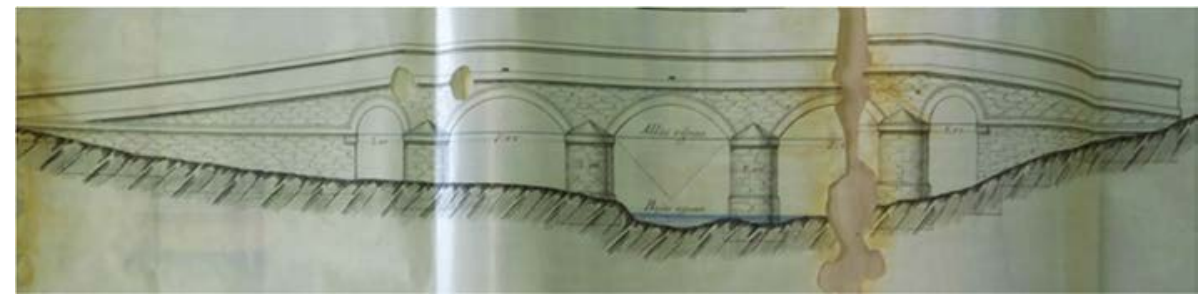

Figura 5. Proyecto de puente sobre el regato Rivera-Aurela, Santiago de Carbajo.

Fuente: Archivo Histórico Provincial de Cáceres. Escala: 1:100

Los pilares proyectados son de sección rectangular terminados en sus lados menores por tajamares semicirculares con sombreretes, como el puente de Santiago de Alcántara. Para Aliseda, Arroyomolinos de Montánchez o Villanueva de la Vera proyectó tajamares en forma de triángulos equiláteros. Estos elementos, colocados con el fin de impedir choques y formación de grandes remansos, se colocarían en un solo lado del puente, pero como se puede observar en las fotografías del Puente del Monte, al igual que en todos los proyectos en estudio, en virtud de la velocidad de las aguas podían producirse remolinos a la salida de las pilas, por lo que al arquitecto siempre le pareció conveniente colocar tajamares a ambos lados, desempeñando el papel propio de los mismos además del de contrafuertes.

La anchura del puente se supone que sería muy similar a los demás estudiados, ya que en todos los proyectos redacta que la misma sería lo suficiente para facilitar el paso de una caballería cargada o de un carro ordinario, dos en el caso del de Pinofranqueado, dejando a cada lado, para la construcción de acitaras o pretiles, un pasillo que oscilaba, según los diferentes proyectos, entre 30 y 50 centímetros. 
En cuanto al material, se puede observar cómo el Puente del Monte está construido, en su totalidad, con sillares de granitos, si bien el relleno seguramente fuese de mampostería. Los demás puentes, bien por no haber piedra granítica cerca o bien por el presupuesto ajustado de las obras, se realizaron de piedra de pizarra y mampostería, encalando algunos detalles como elementos decorativos.

Igualmente, todas las construcciones del arquitecto finalizaban con un pretil con la misma disposición decorativa, tanto en el arranque del mismo, con una hilera que sobresale del plano vertical y en el remate de aquel, como en el sistema de canalización para el desagüe. Además, añadiendo belleza a la obra, se puede observar que las piedras de las impostas, como todo el pretil, aportan un color más claro que el resto. Posiblemente, si se siguió la línea de los otros proyectos, estas partes pudieron estar en algún momento enlucidas.

\section{PROCESO CONSTRUCTIVO DEL PUENTE DEL MONTE.}

El proceso constructivo del puente ${ }^{21}$ se inicia en 1882, cuando el diputado provincial Juan Crisóstomo Gómez solicitó, a la Comisión de Hacienda, 50.000 pesetas para subvencionar la construcción de pontones y la recomposición de caminos de aquellos partidos judiciales más necesitados. Conscientes de que dicha cantidad no supliría en su totalidad el coste de las obras, ello sin embargo ayudaría a paliar la difícil situación económica que los Ayuntamientos estaban atravesando y así poder realizar dichos proyectos.

Para que las solicitudes fueran aceptadas debían acompañarse del correspondiente proyecto desarrollado por una persona facultativa, y una vez expirado dicho término, el Señor Gobernador convocaría a la Diputación a sesión extraordinaria para determinar las obras que se habrían de subvencionar con la cantidad fijada. Dicha reunión fue celebrada el 15 de abril de 1882, y el 1 de mayo del mismo año se redactó una circular anunciada en el Boletín Oficial de la Provincia ${ }^{22}$, recordando que el plazo para la admisión de solicitudes sería hasta el 20 de octubre.

El 1 de junio de 1882 ya se había concedido al pueblo de Ahigal la subvención solicitada para la construcción de un puente sobre el río Alagón, por lo que el alcalde agilizó los trámites administrativos y devolvió a Diputación el proyecto para la instrucción del oportuno expediente.

21 ADPCC, "Subvención para el Puente sobre el río Alagón pedido por el Ayuntamiento de Ahigal. 1882 - 1883" Signatura: 5173.

22 Boletín Oficial de la Provincia de Cáceres, núm. 135, de jueves 11 de mayo de 1882. Circular núm. 14. 
Para justificar aún más la necesidad del levantamiento de esta obra, el 17 de octubre, el Ayuntamiento y la Junta de Asociados redactarían un documento dirigido a la Excelentísima Diputación de Cáceres, en el que se reflejaban los problemas de los que adolecían y la necesidad de construir un puente en proporción y en virtud del caudal de las aguas del Alagón. Exponían que cuando las aguas subían, los vecinos se encontraban separados de sus cultivos por el río, quedando desatendidos; y que al contrario, cuando el nivel de las aguas era demasiado bajo, no podía pasar la barca que utilizaban normalmente, teniendo que paralizar sus trabajos. Igualmente, alguna vez que otra, su ganado perecía por no dar el paso competente para conducirles al pasto necesario para su sustento y las desgracias personales que más de una vez sucedieron, "aun parece resonar todavía los ecos agonizantes de los náufragos implorando socorro"

La urgencia de construir el puente no respondía sólo a la necesidad de los vecinos de Ahigal, sino también a la mayor parte de los de Sierra de Gata, de paso al término de Hurdes hasta la Alberca, o el contacto con Plasencia, ciudad muy ligada al ferrocarril. Había otra necesidad indispensable, la carretera para enlazar las estaciones de Plasencia y Ciudad Rodrigo, siendo el puente un punto de paso.

Por último solicitaban algo más: “. ...y viendo que la Excelentísima Diputación Provincial tienen consignadas en su presupuesto la cantidad de 50.000 pesetas con destino a subvenciones, obras públicas análogas al puente en cuestión, a ella suplicamos y rogamos nos conceda la cantidad del 90\% o en caso de por cuestiones análogas nos concede lo que buenamente pueda..."

Cuando los pueblos cercanos a Ahigal se enteraron de la iniciativa que se estaba llevando a cabo desde ese Ayuntamiento, no dudaron en unirse y dirigirse a Diputación para exponer que no sólo Ahigal se vería beneficiado con ese paso, sino que la mayor parte de la provincia podría obtener beneficios y avances con la nueva construcción. Estos fueron, entre otros: Palomero, Marchagaz, Casar de Palomero, Valdeobispo, Carcaboso, Santibáñez el Bajo, Guijo de Granadilla, Oliva de Plasencia, Riveraoveja, Santa Cruz de Paniagua, Pinofranqueado, Cerezo, Caminomorisco o Nuñomoral. Entre sus instancias estos ayuntamientos sostenían que "más que tratarse de una obra municipal, debería considerarse Obra Provincial" o que la obra proyectada era de "interés general para toda la región derecha del Alagón, por ser el punto único por donde poder comunicarnos con la Ciudad de Plasencia"

El Ayuntamiento de Plasencia también mostró su apoyo. Ramón García, como alcalde, y Pantaleón Iglesias, como representante del sindicato, apuntaban que la importancia de la comunicación entre los pueblos y Plasencia era 
necesaria por motivos comerciales y administrativos, aún más cuando el ferrocarril atravesara el territorio y estos pueblos no tuvieran acceso al mismo.

El proyecto que Ahigal presentaba satisfacía, sin duda, estas condiciones, pero no siendo suficientes sus propios recursos para ejecutar la obra, cuyo presupuesto se había fijado aproximadamente en 80.000 pesetas, y teniendo esta carácter provincial, el Ayuntamiento de Plasencia suplicaba a Diputación en su escrito "se digne a auxiliar la construcción del referido puente del Ahigal con el 90\% del importe de su presupuesto aplicando a esta atención la parte necesaria a los créditos autorizados"

La Comisión de Fomento, tras examinar los antecedentes remitidos por el Ayuntamiento de Ahigal y también de las 17 solicitudes de los pueblos que se habían asociado, consideraron que las formalidades aludidas eran ciertas, y proponía a la Diputación que subvencionara la obra con un importe que, aunque no llegaba al $90 \%$ del coste final, ascendiese a la cantidad de 64.173 pesetas y 97 céntimos, un $80 \%$ aproximadamente.

El resto, que tendría que ser financiado por parte del Ayuntamiento ahigalense, ya había sido fijado en la sesión extraordinaria del 8 de marzo de 1883, que solicitaría del Gobierno la autorización para enajenar cierta clase de valores y retirar de la Caja del Ferrocarril obligaciones. También se acordó quedar exentas del pago a las clases jornaleras.

Por tanto, y debido a que el presupuesto del año corriente ascendía a unos gastos de 12.027 pesetas y 57 céntimos, habría que cubrir tanto estos gastos como la parte no subvencionada del proyecto. Para cubrir esta suma, el pueblo contaba con los productos ordinarios de propios, el producto de la Dehesa Boyal, venta de basura de las callejas públicas y donativos voluntarios, con lo cual, no contando el municipio con más recursos que los consignados, sólo se podía hacer uso para la ejecución de la obra proyectada los siguientes:

"El 18\% del 16 de recargo local sobre inmuebles cuya cantidad se calcula se podrá ascender a dos mil cuatrocientas sesenta y siete con setenta y cuatro céntimos $2.467,74$

El 18\% sobre la contribución milimetral calculada ochenta y cuatro pesetas diez céntimos 84,10

El 70\% sobre el cupo que por causa nos satisfacen al Tesoro esta localidad importante tres mil setecientos tres pesetas veinte y cinco céntimos .........3.703, 25

El 90\% sobre el importe de las cédulas de empadronamiento ciento treinta y nueve pesetas veinticinco céntimos 139,25 
El producto de los títulos de la renta del $4 \%$ perpetuo a interior importante catorce mil pesetas vendidas al 64,30, nueve mil dos pesetas .9 .002

Cuarenta y cuatro obligaciones del Ferrocarril que este municipio tenía consignados en la caja del mismo a quinientas pesetas cada una, veinte y dos mil pesetas 22.000

TOTAL: $37.396,34 ”$

Por tanto, Ahigal contaba con un presupuesto insuficiente para hacer frente a las obras. El Ayuntamiento sacó la obra a subasta pública, de manera que el contratista tendría toda la responsabilidad y saldría la subasta más económica.

Tras un estudio de todas las propuestas realizadas por los diferentes pueblos para la obtención de la subvención, sería el 13 de mayo de 1884 cuando, oficialmente, el Vicepresidente de la Comisión Provincial se dirigiría al Gobierno Civil de la Provincia para remitir una copia de la Resolución de la Diputación Provincial, por la que se acordó aprobar las obras proyectadas para la construcción del puente sobre el río Alagón, a fin de que se uniera al expediente promovido por el Ayuntamiento de Ahigal, con objeto de que se le autorizara la conversión en Títulos al portador de las Inscripciones Intransferibles del 80\% de bienes propios, para proteger, en parte, los gastos que generaran las mencionadas obras.

La Diputación, finalmente, acordó financiar el $50 \%$ de su costo, cuyo presupuesto ascendía a 43.469 pesetas y 21 céntimos, el otro 50\% correspondía al pueblo, que solicitaría la autorización del Gobierno para retirar títulos del 4\% y obligaciones del ferrocarril del Tajo. El aumento en el presupuesto de 6.641 pesetas resultó del coste del proyecto, dando por resultado un nuevo presupuesto de 87.212 pesetas 28 céntimos.

El 28 de mayo, tras haber finalizado el período establecido para la recepción de propuestas, se procedió a la apertura de las dos proposiciones presentadas. Finalmente, la obra recayó en manos de José Lorenzo y Rodríguez, alarife de 49 años, residente en el pueblo vecino de Riolobos, que presentó una oferta para mencionada obra por la cantidad de 86.750 pesetas.

José Lorenzo, bajo compromiso expuesto en la cláusula quinta del contrato, tendría que finalizar la obra de año y medio, a no ser que por cualesquiera divergencia alguna a la voluntad del contratista y previa justificación en este se le conceda prórroga. 
Iniciadas las obras y transcurrido algo más de medio año, se observó cómo el proyecto inicial tenía imperfecciones que obligaban a reformarle, resultando como consecuencia un aumento en el presupuesto de 13.281 pesetas. El Ayuntamiento de Ahigal consideró justo que Diputación concediera el aumento de 6.641 pesetas por el mayor costo debido a un error del proyecto, aunque José Lorenzo firmara en su contrato la cláusula segunda, donde se obligaba a pagar los gastos de escritura y demás gastos que se vean hasta la terminación del contrato.

Tras examinar el proyecto inicial, la Comisión de Fomento consideró justo conceder dicho aumento, así el 28 de abril de 1886 se dio respuesta al Ayuntamiento.

Diputación, realizó un informe sobre el aumento y coste de las mismas, respondió al alcalde de Ahigal diciendo que el arquitecto encargado de la inspección de las obras del puente había recibido su comunicación, en la que se servía ordenarle en forma y razones los aumentos que había tenido la obra.

Como ya se ha mencionado, el inspector encargado de revisar las obras sería Vicente Paredes, pues entre 1870 y hasta su cese en 1894 fue Arquitecto Municipal de Plasencia ${ }^{23}$, partido judicial al que pertenecía Ahigal.

Paredes amparó la responsabilidad moral por el aumento del coste presupuestado por D. Ricardo Morquecho, ayudante de Obras Públicas, salvando sus errores por los datos que le fueron dados, pues el aumento de la obra provenía principalmente de las fundaciones de cimentación. Los aumentos de obra originados por la cimentación fueron causados por errores en la clasificación de las rocas, pues suponiendo que el terreno era de roca dura, su estratificación era suelta y expoliable, incapaces de sostener en ellos el peso de las pilas, originando que la $\mathrm{n}^{\circ} 2$, que estaba proyectada su implantación a $3 \mathrm{~m}$. de altura, había aumentado a 3,60 m. la fábrica de la pila. Otras, al mirlar el cimiento por los puntos más bajos del terreno, habían producido zonas blandas que, mezcladas con partes duras, se habían encontrado en la cimentación. Las causas enumeradas habían producido los aumentos de altura en las pilas y estribos siguientes, enumerados de la orilla oriental a la occidental: en la pila $\mathrm{n}^{\mathrm{o}} 1$ había crecido su altura $40 \mathrm{~cm}$.; en la n $2,3 \mathrm{~m}$. y $60 \mathrm{~cm}$.; en la $\mathrm{n}^{\circ} 3,80 \mathrm{~cm}$.; en la n $7,2 \mathrm{~m}$. y 10 $\mathrm{cm}$.; en la $\mathrm{n}^{\mathrm{o}} 8,90 \mathrm{~cm}$; y en el estribo $\mathrm{n}^{\mathrm{o}} 2,1 \mathrm{~m}$. y $20 \mathrm{~cm}$. Esto, agravado con el olvido de la ubicación de los salmeres de las bóvedas, al duplicarse el

23 DOMÍNGUEZ CARRERO, M., La generación del 98' en la periferia: D. Vicente Paredes Guillén, trabajo inédito, 1990. Archivo Municipal de Plasencia. En PIZARRO GÓMEZ, F.J., Vicente Paredes Guillén y el patrimonio extremeño. Trujillo: Real Academia de Extremadura de las Letras y de las Artes, 2004, p. 14. 
emboquillado, junto con un pequeño error en la cubicación del sillarejo de las bóvedas, habían originado un aumento total en la obra y coste de 47.743 pesetas 18 céntimos.

El 12 de agosto de 1887, Emilio $\mathrm{M}^{\mathrm{a}}$ Rodríguez reconoció las obras ejecutadas. A esa fecha ya estaban construidos los estribos y las pilas, volteados todos los arcos, si bien sin terminar los tres últimos, casi terminados las impostas y preparado el trasdós de las bóvedas para recibir la capa de hormigón.

Confrontadas las partidas de la liquidación, se hallaron conformes con la obra ejecutada, considerando, hasta aquel momento, el valor de la misma en la cantidad de 105.948 pesetas y 39 céntimos, cuyo 50\% importaba a 52.974 pesetas y 19 céntimos, pero habiéndosele satisfecho mediante tres certificaciones anteriores al Ayuntamiento de Ahigal la suma de 42.760,05 pesetas sólo debía percibir por la presente la de 10.214 pesetas y 14 céntimos.

El 12 de octubre, se notificó al arquitecto que inspeccionaba dichas obras que el día 13 de noviembre quedarían terminadas las mismas, lo que le participaba para que dictase las órdenes convenientes para la recepción provisional de dicha construcción. Y así fue, el arquitecto consideró que el proyecto se había realizado en su conjunto ateniéndose a las indicadas consideraciones, aunque hubo alteraciones encaminadas a modificar, con ventaja, el proyecto inicial, levantándose acta en presencia del alcalde, Nicasio García; del secretario, Juan García; de Vicente Paredes y Santos Pulido en representación del contratista.

La comisión de Fomento se hizo cargo de la instancia que el Ayuntamiento de Ahigal dirigió a Diputación para el aumento de la subvención concedida. Dicha instancia iba acompañada del presupuesto adicional realizado por el Arquitecto encargado de la inspección de las obras, el cual ascendía a la suma de 47.743 pesetas y 18 céntimos, cuya mitad, que importaba 23.871 pesetas y 59 céntimos, y que se proponía a Diputación conceder a Ahigal, solicitaban, y que en lo sucesivo no se otorgase por ese concepto otra suma que el 50\% que arrojaba el presupuesto que se acompañaba a la solicitud de subvención. Este dictamen fue emitido en noviembre de 1888 desde el Palacio Provincial, estando presentes Antonio Asensio, Santiago, Juan Muñoz y Enrique Montánchez y aprobado en sesión del día 7 de noviembre por Pedro L., Santiago Antúnez y Agustín Calle, en función de presidente y secretarios respectivamente.

Cuando las obras estaban ultimándose, en 1889, se procedió a la liquidación de las mismas donde hubo un error numérico en un informe emitido por el Ingeniero de la Provincia, de 100 pesetas y 18 céntimos, certificadas de más al contratista. Como consecuencia, en lugar de ser el importe líquido de la obra de 
128.703 pesetas y 19 céntimos, quedaría reducido a 128.602 pesetas y 61 céntimos.

Finalmente, las obras terminaron el 21 de mayo de 1889. Todos los señores que asistieron al acto y contratistas examinaron la obra y comprobaron que en las fábricas no hubo movimientos, ni desperfectos originados por vicios de construcción, únicamente en el terraplén del estribo de la derecha se observó algo de depresión en la rasante, por efecto de la natural consolidación del mismo, quedando el contratista obligado a invertir unas cuantas peonadas en dicho reparo.

No teniendo otra cosa que exponer, se acordó dar por recibida definitivamente la expresada obra ${ }^{24}$.

\section{VICENTE PAREDES GUILLÉN Y SU FUNCIÓN COMO INSPECTOR}

Además de arquitecto, Paredes ${ }^{25}$ fue un destacado intelectual y erudito de la época, como ponen de manifiesto sus investigaciones, su biblioteca, sus escritos y los contactos que mantuvo con destacadas personalidades del mundo de la Historia, la Arqueología, la Cultura y la Ciencia ${ }^{26}$; y ocuparía importantes cargos como Académico correspondiente de la Real Academia de la Historia o como Vocal de la Comisión Provincial de Monumentos Históricos y Artístico de Cáceres $^{27}$.

Centrándonos en lo concerniente a nuestro estudio, en el campo de la ingeniería civil, entre otras materias, Paredes se interesaría por mejorar las condiciones de comunicación de las ciudades por la inminente llegada del

24 ADPCC, "Expediente de la construcción de un puente sobre el río Alagón con subvención de los fondos provinciales. Acta de recepción definitiva", Signatura: 05172, $1887-1892$.

25 Vicente Paredes (1840 - 1916) nació en el municipio de Gargüera (Cáceres). Pasaría los primeros años de su vida en Valdeobispo, localidad en la que Diego Julián Paredes, su padre, trabajó como secretario del Ayuntamiento. A los 28 años, Vicente finalizaría sus estudios de Arquitectura en la Escuela Especial de Arquitectura de Madrid y, antes de finalizar el año, ya ocuparía, de manera provisional, la plaza de Arquitecto Provincial de Cáceres. Por motivos familiares se trasladaría a Plasencia, ciudad donde desarrolló gran parte de su actividad arquitectónica. En DOMÍNGUEZ CARRERO, M.M., Vicente Paredes Guillén: biografia, Cáceres, 2006, pp. 61 y ss.; como arquitecto municipal y diocesano. En HURTADO URRUTIA, M., "Siluetas desde el olvido. Vicente Paredes Guillén (1840-1916)", Hoy, 28 de enero de 1996, p. 31. En PIZARRO GÓMEZ, F.J., Vicente Paredes Guillén y el patrimonio extremeño. Trujillo: Real Academia de Extremadura de las Letras y de las Artes, 2004, p. 14.

26 PIZARRO GÓMEZ, F.J., Vicente Paredes Guillén y el patrimonio extremeño. Trujillo: Real Academia de Extremadura de las Letras y de las Artes 2004, pp. 13-16.

27 HERNÁNDEZ MARÍN, C., "Vicente Paredes Guillén (1840-1916): medio siglo de trabajos, estudios y opiniones sobre la Vía de la Plata". En XIV Jornada de Historia de Fuente de Cantos: La vía de la Plata y otros estudios sobre Extremadura. Lucerna Asociación Cultural de Fuente de Cantos, Badajoz, 2013, pp. 110-129. 
ferrocarril. En la década de 1880, mientras trazaba proyectos como el del alcantarillado o la reforma urbana de Plasencia, mediante la apertura de una calle, paralelamente sería nombrado inspector encargado de las obras del puente en estudio, pues aunque no se conserva el proyecto, se observará cómo durante el proceso constructivo del mismo aparecen repetidas veces su rúbrica, además de haberse localizado un cuaderno de notas donde Paredes recogió, con mimo, el avance de dichas obras durante las inspecciones ${ }^{28}$ que realizó en los años de construcción $^{29}$. Además de trabajar en el ámbito civil, el arquitecto llevaría a cabo numerosas obras religiosas, como las iglesias de San Juan Bautista y Santa María de Don Benito ${ }^{30}$, la iglesia parroquial de Santa Amalia o el Convento de las Carmelitas de Plasencia.

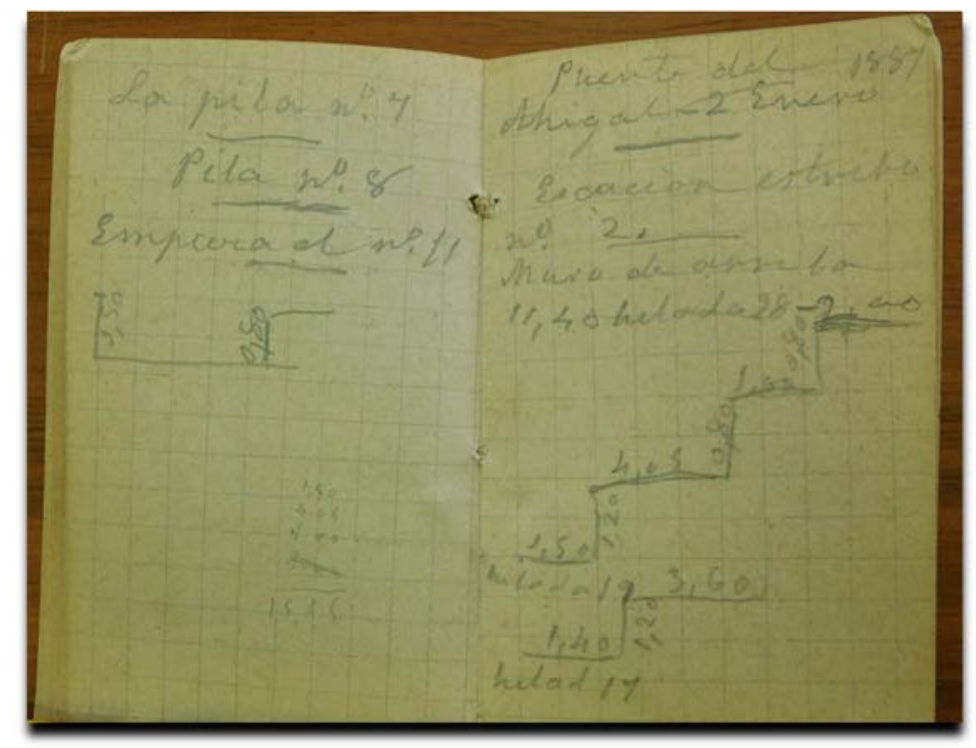

Figura 6. Anotaciones de Vicente Paredes. Detalles del reconocimiento realizado sobre el Puente de Ahigal el 2 de enero de 1887. Fuente: Legado Vicente Paredes, Archivo Histórico Provincial.

28 Las inspecciones fueron llevadas a cabo los días: 13 de febrero y 22 de octubre de $1885 ; 5$ de febrero, 22 de marzo, 1 de mayo, 14 de julio, 13 de agosto y 13 de septiembre de 1886; 2 de enero, 13 de marzo, 13 de abril, 13 de junio y 13 de agosto de 1887 .

29 AHPCC, "Legado Paredes" (38-71) 93.

30 RAMOS RUBIO, J. A., "El arquitecto Vicente Paredes, representante del eclecticismo extremeño, arquitecto en la construcción de la iglesia parroquial de Santa María de Don Benito." En Boletín de la Real Academia de Extremadura de las Letras y las Artes, n² 21, 2013, pp. 667-684. 


\section{CONCLUSIÓN}

Para concluir, y en base a toda la documentación analizada, sostenemos que la obra en estudio es labor de Emilio $\mathrm{M}^{\mathrm{a}}$ Rodríguez, pues en el momento de su proyección, aquel, como arquitecto provincial, llevaría a cabo numerosos proyectos para el desarrollo de las obras públicas en pequeñas localidades de la provincia cacereña, carentes de arquitectos municipales. Además, se percibe una gran similitud en las estructuras arquitectónicas, materiales y elementos decorativos de los proyectos estudiados.

El puente en estudio supuso un gran avance para la población de Ahigal y la de muchos otros pueblos cercanos que vieron como, a partir de su construcción, desaparecían todos los problemas de los que habían adolecido hasta el momento; nos referimos a la imposibilidad de vadear el cauce del río durante largos períodos de tiempo, que, a su vez, impedía el flujo comercial con la importante localidad cercana de Plasencia.

Por otra parte, gracias al rico legado conservado de Vicente Paredes conocemos en profundidad su trayectoria, y nunca planificó una obra de ingeniería civil de estas características, pero por su formación, experiencia y cercanía al lugar, en el momento del levantamiento del puente, no habría personalidad más cualificada que él para llevar la inspección de unos trabajos que ascendieron, inicialmente, a una cuantía aproximada de 80.000 pesetas y que terminaron siendo, debido a varios contratiempos durante la ejecución de las mismas, de 128.602 pesetas y 61 céntimos, costeados al $50 \%$ por Diputación de Cáceres y por el pueblo de Ahigal.

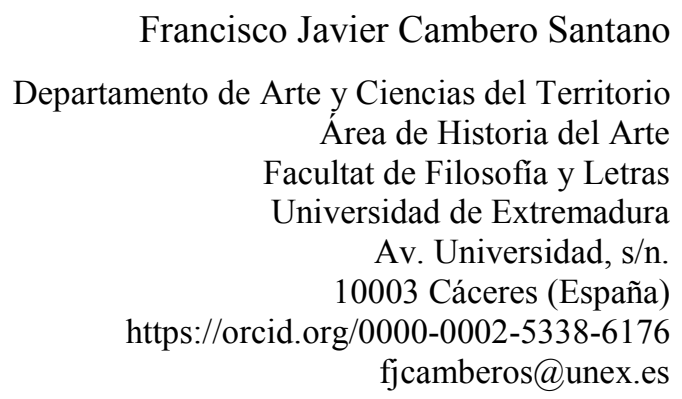

